\title{
Flexible reproductive timing can buffer reproductive success of Pygoscelis spp. penguins in the Antarctic Peninsula region
}

\author{
Jefferson T. Hinke ${ }^{1,2, *}$, Michael J. Polito ${ }^{3}$, Christian S. Reiss ${ }^{1}$, Susan G. Trivelpiece ${ }^{1}$, \\ Wayne Z. Trivelpiece ${ }^{1}$
}

\begin{abstract}
${ }^{1}$ Antarctic Ecosystem Research Division, Southwest Fisheries Science Center, National Marine Fisheries Service, National Oceanic and Atmospheric Administration, 3333 North Torrey Pines Court, La Jolla, California 92037, USA

${ }^{2}$ Scripps Institution of Oceanography, University of California San Diego, 9500 Gilman Drive, La Jolla, California 92093, USA

${ }^{3}$ Department of Biology and Marine Biology, University of North Carolina Wilmington, 601 South College Drive, Wilmington, North Carolina 28403, USA
\end{abstract}

\begin{abstract}
Varying the timing of reproduction in response to local environmental conditions is a key factor influencing reproductive success. We used $19 \mathrm{yr}$ (1991 to 2009) of data on breeding chronology and reproductive success of Adélie penguins Pygoscelis adeliae and gentoo penguins P. papua at King George Island, Antarctica to compare clutch initiation dates (CIDs), hatch rates, and crèche rates at population and individual levels. The CIDs of both species occurred earlier with warmer October temperatures, but gentoo penguins adjusted CIDs twice as much per ${ }^{\circ} \mathrm{C}$ of temperature as Adélie penguins. Gentoo penguins also exhibited lower variances in hatch and crèche rates than Adélie penguins, suggesting that greater ability to respond to ambient conditions can buffer reproductive success. Mixed-effects models revealed that individual- and yearspecific random effects on the overall population mean best accounted for observed variation in CIDs, with some individuals routinely breeding earlier than others. However, individual differences in the relative timing of breeding provided no advantage for long-term reproductive success. The results suggest that, among gentoo and Adélie penguins, plasticity in CIDs with respect to environmental conditions is primarily a population-level rather than an individual-level response. Energetic constraints of contrasting migratory and fasting behaviors likely contribute to the different abilities to respond to warming spring temperatures. Overall, larger responses of gentoo penguin CIDs to October temperature and lower variability in reproductive success, despite reduced reproductive success with delayed breeding, suggests that gentoo penguins are better equipped than Adélie penguins to adjust to rapid warming in the Antarctic Peninsula ecosystem.
\end{abstract}

KEY WORDS: Phenology $\cdot$ Adélie $\cdot$ Gentoo $\cdot$ Climate change $\cdot$ Migrant

Resale or republication not permitted without written consent of the publisher

\section{INTRODUCTION}

Under changing environmental conditions, species or individuals that exhibit a greater range in phenotypic responses (plasticity) may exhibit improved reproductive success and, ultimately, higher fitness (Stearns 1989, Visser 2008). Among birds, varying the timing of breeding according to local environmental conditions has long been understood as important for reproductive success, where aligning food demand for offspring with peak food availability is critical for successful chick production (Perrins 1970). Species or individuals with the ability to adjust breeding chronology toward optimal conditions 
should exhibit higher and/or less variable reproductive success relative to those less able to respond to ambient conditions. In the present study, we compared the response in breeding chronology to changing environmental conditions in the Antarctic Peninsula region of 2 species of Pygoscelis penguins that have exhibited long-term divergent trends in abundance. We specifically investigated the relative importance of population- versus individual-level plasticity on reproductive success among Adélie penguins $P$. adeliae and gentoo penguins $P$. papua.

The Antarctic Peninsula region is currently undergoing rapid environmental changes, including increased air and sea temperatures (Meredith \& King 2005, Turner et al. 2005a), declines in the duration of winter sea ice (Stammerjohn et al. 2008), and increases in frequency and amount of precipitation (Turner et al. 2005b, Thomas et al. 2008). These environmental factors are important for penguins, as they can influence marine primary production (Schofield et al. 2010), food distribution (Loeb et al. 1997), and physical conditions of nesting sites (Patterson et al. 2003, Boersma 2008). In particular, access to snowfree ground is crucial for reproductive success among the ground-nesting Pygoscelis spp. penguins (Patterson et al. 2003, Boersma 2008). During spring, cold conditions that prevent melting of ice and snow or large snowstorms that bury nesting areas may delay nest-building efforts. Thus, local weather conditions may directly impact the timing of reproduction in penguins. Evidence from multiple breeding colonies in the northern and western Antarctic Peninsula region support this assertion; delayed clutch initiation dates (CIDs) for Adélie and gentoo penguins correspond with colder spring temperatures (Lynch et al. 2009, 2012, this volume).

Irrespective of local environmental conditions, species may differ in their ability to alter breeding chronology. Lynch et al. $(2009,2012)$ reported that gentoo penguins were capable of advancing mean egg-laying dates with respect to mean October temperatures roughly twice as much as Adélie penguins (3.2 versus $1.7 \mathrm{~d}^{\circ} \mathrm{C}^{-1}$ ) in the Antarctic Peninsula region. The different responses to spring temperatures may arise because of contrasting energetic constraints that are dictated by species-specific migratory behaviors (Both et al. 2010). Adélie penguins return to breeding colonies after migrating from distant wintering habitats, build and occupy nest sites, all the while fasting for the $3 \mathrm{wk}$ duration of courtship and egg-laying (Ainley et al. 1983). Following clutch completion, males remain on the nest to incubate the eggs, on average, for an additional 2 wk (Trivelpiece
\& Trivelpiece 1990). The migration and month-long breeding fast of Adélie penguins is in contrast to gentoo penguins, which typically forage in the near-shore waters year-round (Clausen \& Pütz 2003, Tanton et al. 2004). The female gentoo may fast for up to a week before egg-laying, but thereafter resumes daily foraging trips, with frequent incubation exchanges with the male (Trivelpiece \& Trivelpiece 1990). Hence, gentoo penguins have neither a pre-breeding migration nor the requirement to fast for prolonged periods during the breeding season. Furthermore, the more local distribution of gentoo penguins during winter may provide them with better information about nesting conditions and facilitate timely decisions about when to initiate breeding relative to a long-distance migrant with no knowledge of local conditions prior to arrival (Both et al. 2010). Without local knowledge of nesting conditions prior to arrival in the nesting colony, Adélie penguins may have limited ability to alter their breeding chronology to match prevailing conditions.

Despite apparently different capabilities to adjust breeding chronology in response to ambient conditions (Lynch et al. 2009, 2012), it remains unclear how reproductive success of Adélie and gentoo penguins might be affected on both population and individual levels. In the South Shetland Islands, data from $24 \mathrm{yr}$ of observational studies suggested that the fledging rates of Adélie penguins were positively correlated with fledging rates of gentoo penguins (Hinke et al. 2007). A positive correlation between species suggests that reproductive success of both species is constrained by local conditions (e.g. snow and ice cover, food availability, predation) during the nesting period. However, the positive correlation does not reveal the magnitude of variation in reproductive success or whether different abilities to vary breeding chronology are related to the magnitude of variation in reproductive success. In the present study, we investigated the relationship between variation in the timing of egg-laying and variation in reproductive success on a population level. Our guiding assumption was that plasticity in breeding phenology ought to reduce variability in breeding success. Thus, a greater ability of a species or an individual to adjust breeding chronology under a broad range of environmental conditions ought to result in reproductive success that exhibits lower variation relative to its mean level. On a population level, we asked whether a greater ability to vary the timing of egglaying with respect to local environmental conditions results in more stable hatch and crèche rates 
over a range of environmental conditions. On an individual level, we asked whether differences in individual responses to environmental conditions are related to long-term reproductive success (LRS). In particular, we assumed that less ability to adjust the timing of egg-laying may result in breeding while nesting conditions are poor, leading to increased risk of poor reproductive success (Monrós et al. 1998).

Based on the regional correlations of breeding chronology with mean October temperatures (Lynch et al. 2009, 2012), we examined variation in egglaying dates with respect to this environmental variable. We first examined the extent to which the breeding chronology of Adélie and gentoo penguins varies with respect to ambient temperatures prior to the breeding season, and then we related variation in breeding chronology to variation in reproductive success. We hypothesized that, at a population level, greater interannual variation in breeding chronology is associated with lower interannual variation in hatching and crèching rates. Secondly, we hypothesized that greater plasticity on an individual level increases LRS.

\section{MATERIALS AND METHODS}

\section{Study site}

We examined data on breeding chronology and reproductive success from long-term monitoring studies of Adélie and gentoo penguins at the Copacabana colony in Admiralty Bay, King George Island $\left(62^{\circ} 10^{\prime} \mathrm{S}, 58^{\circ} 30^{\prime} \mathrm{W}\right)$. The Copacabana colony, a mixed colony within which the Adélie and gentoo penguins breed, is located near the northern limit of the Adélie penguin's breeding range, but near the southern limit of the gentoo penguin's breeding range (Trivelpiece et al. 1987). Nesting locations in the study colony are located on the rocky beaches adjacent to Admiralty Bay, and on the wind-swept northwest-facing slopes above the beaches. Typically, Adélie penguins return to the colony in early to mid-October, usually prior to our arrival in the camps, and breeding begins by late October. Gentoo penguins remain near the colony yearround and typically initiate breeding shortly after the onset of breeding by Adélie penguins. From 1991-92 to 2009-10, the colony of Adélie penguins declined from 5721 to 2102 breeding pairs, while the gentoo colony grew from 2261 to 3755 breeding pairs.

\section{Reproductive study}

To assess population-level variability in the annual means for CIDs, hatching rates, and crèching rates, we used reproductive-success data collected during 19 austral summers (1991-92 to 2009-10) for Adélie and gentoo penguins. The data used here derived from 20 to 40 sets of 5 adjacent nests (nest groups) that are distributed throughout the colony. The larger sample size (200 nests $\mathrm{yr}^{-1}$ ) was collected in the first year of the study, but was reduced to 100 nests starting in 1992-93 to accommodate declining population sizes of Adélie penguins. Each year, new nest groups with un-banded birds were chosen for study. The selection of new nest groups every year was intended to provide an estimate of population productivity that was not biased by demographic changes in reproductive success associated with changes in age or breeding experience of a set of focal individuals. Hereinafter, this annual study will be referred to as the 'reproductive study'. Once nest groups were selected, the reproductive-study birds were permanently marked with uniquely numbered metal flipper bands. In the reproductive study, both mates of Adélie penguin pairs were banded. For gentoo penguins, only 1 mate from each pair was banded and a 1:1 sex ratio was typically achieved each year. The different banding protocols on each species stem from early observations of frequent divorce among gentoo penguins if both mates are banded; similar effects of banding on the divorce rate among Adélie penguins have not been observed (W. Z. Trivelpiece pers. obs.). The birds in the reproductive study were monitored for breeding success in one season only; the flipper bands were left on birds, however, and those birds were then included in studies of adult survival (data not analyzed here).

\section{Non-disturbance study}

To ensure valid comparisons of reproductive success between species with different banding treatments in the reproductive study, we compared data from the reproductive study with data from an analogous study of un-banded birds that are monitored as part of annual non-disturbance studies. Each year in the non-disturbance study, between 7 and 40 groups of 5 nests for Adélie and gentoo penguins were chosen in the same manner as described in the previous subsection for the reproductive study. Non-disturbance birds were neither banded nor handled at any time during the breeding season. Non-disturbance 
data for gentoo penguins were not collected in 1992-93, so comparisons between the reproductive and non-disturbance studies for gentoo penguins are based on 18 yr of data.

\section{Known-age study}

Data on variation in reproductive timing and success at an individual level derive from observations of known-age birds that were monitored across multiple years (hereinafter called the 'known-age study'). Known-age individuals were marked with a uniquely numbered metal flipper band as chicks prior to fledging. All known-age individuals that returned to the natal colony to breed were monitored during all subsequent breeding attempts. To examine individual variation in CID as a function of environmental conditions, we used only data from individuals of breeding age ( $\geq 3 \mathrm{yr}$ old) that initiated a minimum of 3 clutches during a $20 \mathrm{yr}$ study period (1990-91 to 2009-10). This restriction resulted in 69 Adélie penguins that bred between 3 and 9 times each (mean: 4.14 breeding attempts) and 152 gentoo penguins that bred between 3 and 13 times (mean: 5.34 breeding attempts).

\section{Breeding chronology and reproductive success}

In the reproductive, known-age, and non-disturbance studies, each nest was monitored from egglaying until successful crèche or nest failure, whichever occurred first. A successful crèche was defined at the first observation of unattended chicks in the study nest, which typically occurred at a chick age of 20 to $30 \mathrm{~d}$. Monitoring of the nest and chicks ceased at this time because unattended chicks quickly joined other unattended chicks in mobile groups, rendering identification of individual chicks and study nests impossible without the use of additional individually identifiable marks, which are not used on the chicks from the reproductive study. For each nest, the number of eggs laid, chicks hatched, and chicks crèched was recorded. Egg-laying dates were determined via daily visual observations of nest contents, using binoculars or the naked eye, depending on the location of the nest within the study colony. Once the clutch was completed, nest contents were visually verified on $4 \mathrm{~d}$ intervals to minimize disturbance. Once hatching began, daily verification of nest contents resumed until all chicks had hatched. Thereafter, daily observations of nest occupation and verification of nest contents on $4 \mathrm{~d}$ intervals were continued until successful crèche or nest failure. To limit researcher presence near non-disturbance sites, determination of egg-laying dates were not included as part of the non-disturbance study.

For the reproductive study, yearly means for all breeding parameters were calculated as grand means of the averages from each nesting group. Mean CIDs were based on the lay dates of the first eggs laid per nest. Hatching and crèching rates were calculated on a per-nest basis and also on a per-egg or per-hatch basis. The per-nest metric provided an estimate of overall egg and chick production within the colony, while we used the latter metrics to examine offspring survival during the pre-hatch and post-hatch periods.

\section{Individual CIDs and LRS}

From the known-age study, CIDs for each individual were taken as the date of first egg lay in each year that a clutch was initiated. We calculated LRS for each individual as (1) total number of chicks hatched per total number of eggs laid, (2) total number of chicks crèched per total number of chicks hatched, and (3) total number of chicks crèched per total number of eggs laid. The 3 individual-level LRS metrics were used to assess whether hatching rates, chick survival rates during the brood period, and total chick production were sensitive to individual responses to variable environmental conditions. We note that the LRS metrics are not estimates of lifetime reproductive success, because some animals may have shed bands or emigrated from the natal colony, thus securing further breeding opportunities than those recorded.

\section{October temperatures}

Numerous environmental variables may correlate with annual indices of breeding success in penguins. Here, we include only temperature during October, given previous findings of its high correlation with CID within the suite of pygoscelid penguins throughout the Antarctic Peninsula region (Lynch et al. 2009, 2012) and its role in determining conditions in the breeding colony (discussed in 'Introduction'). We used mean October temperatures collected at Bellingshausen Station, located approximately $22 \mathrm{~km}$ west of the study colonies. Mean monthly temperature data, calculated from daily averages of 6-hourly 
observations (V. Lagun pers. comm.), were acquired online from the Russian Antarctic Expedition (www. aari.aq). On the scale of monthly averages, we assumed that spatial differences in mean temperatures over the $22 \mathrm{~km}$ separating the study site and the meteorological station were likely to be small.

\section{Statistical analysis}

To test for the presence of different effects of banding treatments on data in the reproductive study, we used ANOVA to compare the effects of banding (bands present or absent) by species (1 or 2 bands nest $^{-1}$ ) on hatching and crèching rate. A significant interaction between band presence and species would indicate that banding effects differed by species.

We used correlation analysis to compare the breeding chronology and reproductive success for each species. We used $t$-tests to test null hypotheses of no difference between species in the loss rates of eggs and chicks from nests. Because early and late CIDs can be associated with reduced reproductive success (Perrins 1970), we fit quadratic regressions to estimate the effect of yearly mean population CIDs on mean chick production rates per nest. We also compared the quadratic fits to simple linear regression using a likelihood ratio test to assess model parsimony. To compare interannual variation in the reproductive success between species, we computed coefficients of variation (CVs). For hatching and crèching rates, we corrected the CVs for the maximum possible value of data bounded by 0 and 1, as suggested by Morris \& Doak (2004). Throughout, we report means and 95\% confidence intervals unless otherwise noted.

\section{Individual plasticity and LRS}

To estimate individual-level plasticity in CIDs with respect to October temperatures, we fit linear random-effects models to within-subject centered data to differentiate individual-level responses from population-level responses following the methods of van de Pol \& Wright (2009). Briefly, we included withinindividual centered October temperatures, calculated as $\left(x_{i j}-X_{j}\right)$, sex, and either breeding age or breeding experience in an initial model of fixed effects. Here, $x_{i j}$ is the October temperature experienced by individual $j$ in year $i$ and $x_{j}$ is the mean October temperature experienced by individual $j$. We considered age and breeding experience separately to determine which single factor to include in the model, noting that reproductive success during the first breeding attempt, irrespective of age, is typically lower than subsequent attempts (Ainley et al. 1983). Therefore, experience was quantified as a binary factor, indicating either the first breeding attempt or any subsequent attempt. The full fixed-effect model therefore had the generic form:

$$
y=\beta_{0}+\beta_{1}\left(x_{i j}-\bar{x}_{j}\right)+\beta_{2} \bar{x}_{j}+\beta_{3} \operatorname{Sex}+\beta_{4} Z+\varepsilon_{i j}
$$

where $y$ is the estimated CID, $\beta_{0}$ is the fixed-effect intercept, $\beta_{1}$ is the fixed effect for the individualcentered temperature, $\beta_{2}$ is the fixed effect for the mean temperature experience by each individual, $\beta_{3}$ is the fixed effect for sex, coded as a binary dummy variable to test for differences between sexes, $\beta_{4}$ is the fixed effect for either age or breeding experience, $Z$, and $\varepsilon_{i j}$ are normally distributed random errors. We fit the 2 candidate global models and then selected the best model for fixed effects based on Akaike's information criterion (AIC).

Once the fixed effects for each species were specified, we followed the general protocol of Zuur et al. (2009) to identify the most parsimonious full model (fixed + random effects). All models included a random effect for breeding year $Y_{i}$ so that the generic form of the random-effects model was:

$$
\begin{aligned}
y & =\left(\beta_{0}+b_{j}\right)+\left(\beta_{1}+a_{j}\right)\left(x_{i j}-\bar{x}_{j}\right)+\beta_{2} \bar{x}_{j} \\
& +\beta_{3} \operatorname{Sex}+\beta_{4} Z+Y_{i}+\varepsilon_{i j}
\end{aligned}
$$

where $b_{j}$ and $a_{j}$ are the random effects on the intercept and slope respectively for each individual. We then compared 3 models with alternative combinations of the random effects components. First, we examined a model with a random intercept term for each individual $\left(b_{j}\right)$ to test whether CIDs responded in the same way to temperature across individuals, but with some individuals consistently initiating clutches earlier or later than others. The second model evaluated whether all individuals exhibited the same average CID but responded differently to the variation of temperature (i.e. different slopes; $a_{j}$ ). Finally, we examined a model with both a random intercept and random slope for each individual $\left(b_{j}\right.$ and $a_{j}$ ). In this configuration, the models with random slope components can be interpreted as evidence for individual-level plasticity in response to October temperatures, while the model with a random effect on the intercept only would indicate shared rates of response to October temperatures across all individuals, but that individuals exhibited consistent differences in the relative timing of breeding. The model with the lowest AIC score was selected for further 
analysis. As a final step in the model selection process, we used likelihood ratio tests to refine the fixedeffect components of the model and dropped terms that did not improve model performance. After unimportant fixed effects were removed, the final model was refit using restricted maximum likelihood (REML) (Zuur et al. 2009). The random-effects models were fit with the lme4 package (Bates et al. 2011) developed for R statistical software (R Development Core Team 2010). The estimated random effects that best accounted for individual differences in CID were then correlated with 3 indices of LRS (hatches per egg laid, crèches per egg hatched, and crèches per egg laid) to examine whether reproductive success improved with earlier individual CIDs or greater individual-level plasticity.

\section{RESULTS}

\section{Reproductive study}

Comparisons of mean reproductive success data from banded and un-banded birds indicated no evidence for an interactive effect of banding treatment and species on hatch rates $\left(F_{1,73}=0.14, \mathrm{p}=0.71\right)$ or crèche rates (ANOVA: $F_{1,73}=0.53, \mathrm{p}=0.47$ ). However, a negative effect, $\delta$, of banding on hatch rates $\left(\delta=-0.07 \pm 0.06\right.$ chicks nest $\left.^{-1}\right)$ and crèche rates $\left(\delta=-0.10 \pm 0.06\right.$ crèches nest $\left.^{-1}\right)$ was found. Because

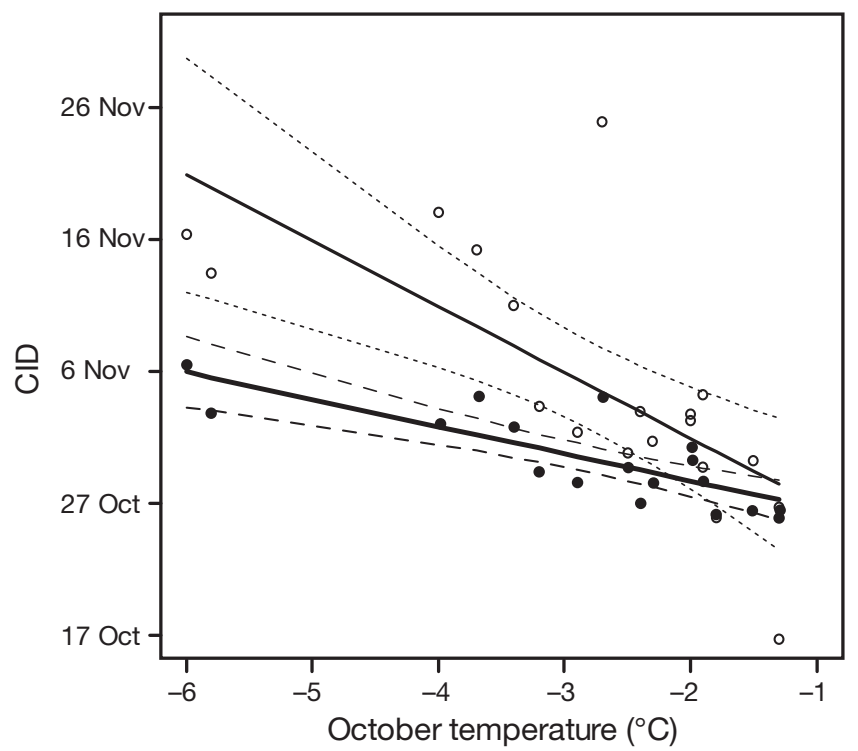

Fig. 1. Pygoscelis adeliae and P. papua. Relationship of mean yearly clutch initiation date (CID) for Adélie (•) and gentoo penguins (o) with mean October temperature. Solid lines are linear regressions with confidence intervals plotted as dashed lines the negative effect of banding was independent of the different band treatments, we proceeded with comparisons using data on hatch and crèche rates from the reproductive study to retain consistency in the data from CID through the crèche period by using the same study birds.

The mean yearly CIDs for both species were positively correlated $\left(r=0.88, t_{17}=7.8, p<0.01\right)$, but the range of CIDs spanned $>1$ full month $(16$ October to 24 November) for gentoo penguins and $<2 \mathrm{wk}$ (25 October to 6 November) for Adélie penguins. On average, gentoo penguins initiated clutches $5.5 \pm$ $3.2 \mathrm{~d}$ after Adélie penguins $\left(t_{18}=-3.6, \mathrm{p}=0.002\right)$, but preceded the mean CID of Adélie penguins on one occasion by $10 \mathrm{~d}$ in 2008-09. The mean yearly CIDs for both species were linearly related with mean October temperatures (Adélie: $\mathrm{R}^{2}=0.66, F_{1,17}=32.4$, $\mathrm{p}<0.01$; gentoo: $\left.\mathrm{R}^{2}=0.50, F_{1,17}=17.0, \mathrm{p}<0.01\right)$, whereby CIDs occurred later in the year under colder October conditions (Fig. 1). The rate of change in the mean population CID was $2.92 \pm 2.5 \mathrm{~d}{ }^{\circ} \mathrm{C}^{-1}$ greater for gentoo penguins than for Adélie penguins $(t=$ $-2.3, \mathrm{p}=0.03$ ), suggesting that gentoo penguins, at a population level, exhibited greater responses in CID to changing October temperatures.

Loss rates of eggs per nest during incubation were higher than the loss rates of chicks per nest for both species (Fig. 2a), suggesting that mortality during the egg stage played a dominant role is shaping overall reproductive success. Moreover, the loss rates of eggs and chicks were greater for Adélie than for gentoo penguins. Hatching rates per nest were positively correlated with the crèche rates per nest for both species (Adélie: $\mathrm{r}=0.93, \mathrm{p}<0.01$; gentoo: $\mathrm{r}=0.98, \mathrm{p}<$ 0.01; Fig. 2b), but the correlations between offspring survival during the incubation period (hatches per egg) and brood period (crèches per hatch) were not correlated (Adélie: $r=0.13, p=0.57$; gentoo: $r=0.40$, $\mathrm{p}=0.09$; Fig. 2c). This suggests that post-hatch chick survival was relatively independent of the conditions that contributed to variable hatching rates.

At the population level, Adélie and gentoo penguins exhibited contrasting patterns of variability in the timing of breeding relative to variability in reproductive success. Adélie penguins exhibited lower interannual variation in mean CIDs, but higher variation in hatch and crèche rates than gentoo penguins (Fig. 3). In particular, the CV for CID was 3 times as large for gentoo penguins (0.03) as for Adélie penguins $(0.01$; Fig. 3a). The $\mathrm{CV}$ for hatch rates $(0.28)$ and crèche rates $(0.16)$ of gentoo penguins were, respectively, $22 \%$ and $25 \%$ lower than the $\mathrm{CV}$ for hatch (0.36) and crèche rates $(0.22)$ of Adélie pen- 

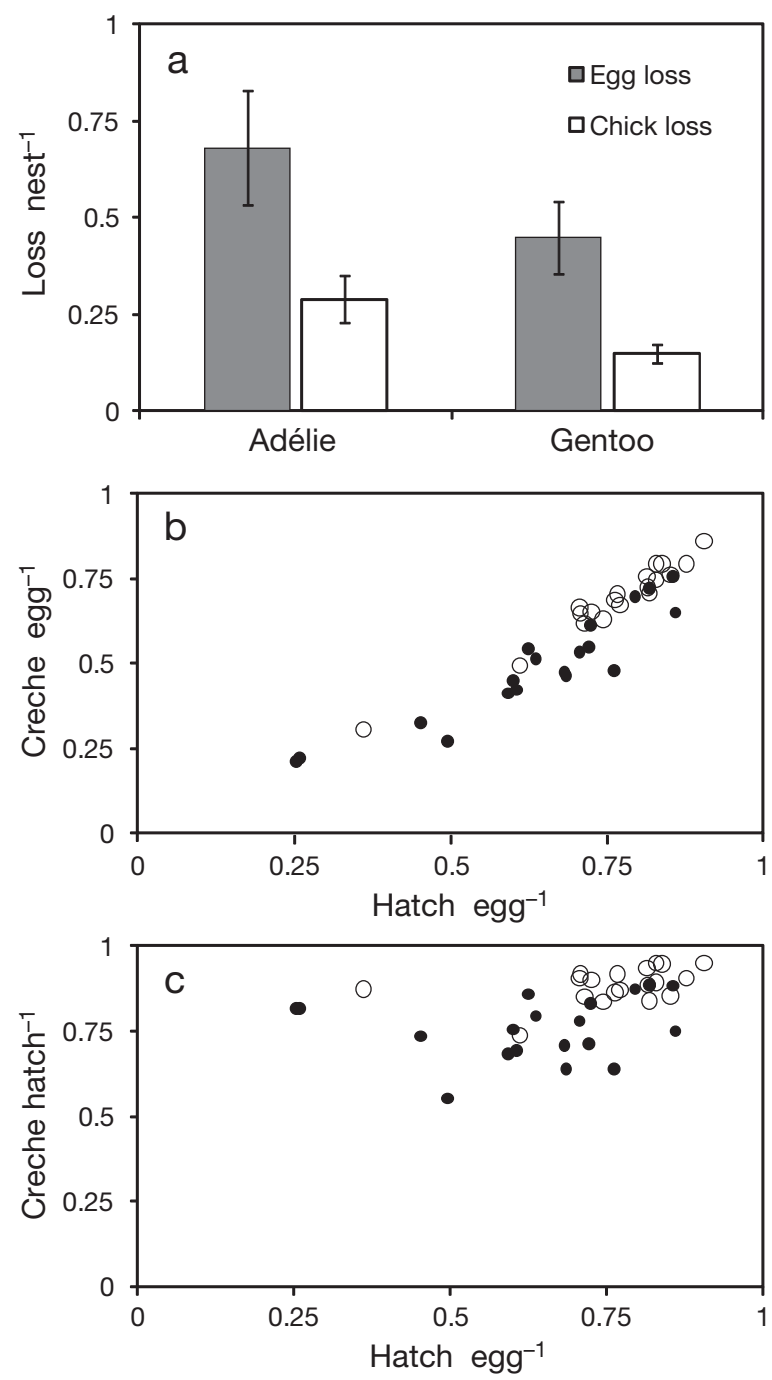

Fig. 2. Pygoscelis adeliae and P. papua. (a) Comparison of mean $( \pm 95 \% \mathrm{CI})$ loss rates of eggs per nest and chicks per egg laid, (b) relationship between annual means of eggs hatched per egg laid and mean number of chicks crèched per egg laid, and (c) relationship between annual means of eggs hatched per egg laid and mean number of chicks crèched per egg hatched, for Adélie $(\bullet)$ and gentoo penguins ( $(0)$

guins (Fig. 3b,c). This suggests that a more flexible population-level response of CIDs to October temperatures may result in greater stability of population-level reproductive success over time.

The species-specific responses of population-level reproductive success to variation in CIDs differed, however. For Adélie penguins, hatches per egg laid and crèches per egg laid exhibited no significant trends with CID (Fig. 4), but the number of crèches per egg hatched was best explained by a quadratic trend $\left(R^{2}=0.30, F_{2,16}=3.5, p=0.05\right.$; Fig. $\left.4 b\right)$, suggesting that post-hatch chick survival declined when breeding occurred too early or late. For gentoo pen-

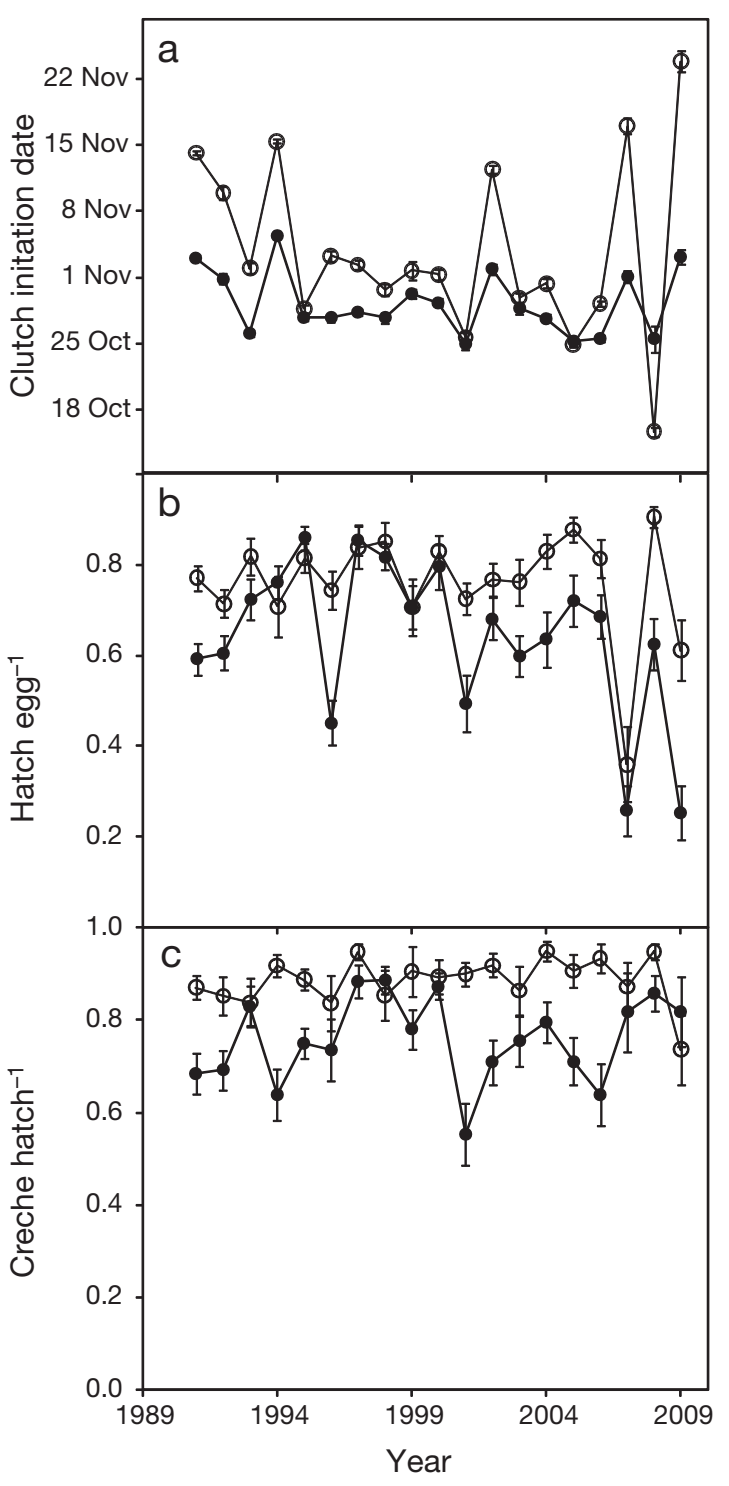

Fig. 3. Pygoscelis adeliae and P. papua. Breeding chronology and reproductive success parameters for Adélie $(\bullet)$ and gentoo penguins (O). Yearly means $( \pm \mathrm{SE})$ of (a) clutch initiation date (CID), (b) hatching success, and (c) chick survival

guins, linear trends were evident in all metrics of reproductive success (hatches per egg: $\mathrm{R}^{2}=0.48, F_{1,17}=$ 15.5, $\mathrm{p}<0.01$; crèches per hatch: $\mathrm{R}^{2}=0.29, F_{1,17}=6.9$, $\mathrm{p}=0.02$; crèches per egg: $\mathrm{R}^{2}=0.53, F_{1,17}=19.7, \mathrm{p}<$ 0.01; Fig. 4). Despite the trends for improved reproductive success with earlier breeding among gentoo penguins across all metrics, the quadratic relationship of post-hatch chick survival among Adélie penguins indicated that variation in CID does affect reproductive success. The greater variation in hatching rates across CIDs for Adélie penguins, however, appeared to mask the affect of the quadratic trends in posthatch survival rates on total crèche rates. 


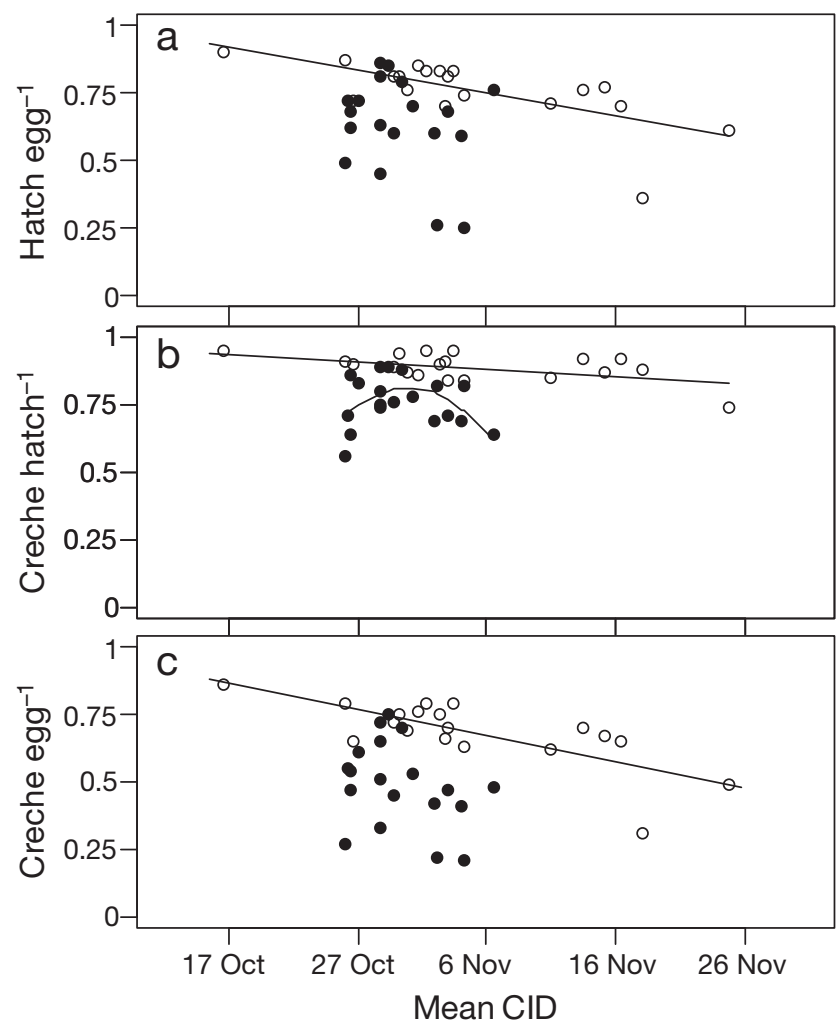

Fig. 4. Pygoscelis adeliae and P. papua. Relationship of (a) hatch rates per egg laid, (b) crèche rates per chick hatched, and (c) crèche rates per egg laid to the mean annual clutch initiation date (CID) for Adélie $(\bullet)$ and gentoo penguins (O). Significant $(p \leq 0.05)$ trend lines are plotted
Table 1. Pygoscelis adeliae and P. papua. Comparison of candidate global models for fixed effects of temperature, age, and sex on clutch initiation date in Adélie and gentoo penguins. AIC: Akaike's information criterion; T: mean October temperatures including within-individual centered data $\left(x_{i j}-\bar{X}_{j}\right)$ and mean October temperature for each individual $\left(\bar{x}_{j}\right)$; A: age at breeding; E: breeding experience; S: sex of the breeding bird

\begin{tabular}{|lcccc|}
\hline Fixed-effect model & AIC & df & $\Delta$ AIC & AIC weight \\
\hline Adélie & & & & \\
T + S + E & 1666.6 & 6 & 0.00 & 0.94 \\
T + S + A & 1672.1 & 6 & 5.5 & 0.06 \\
Gentoo & & & & \\
T + S + E & 4719.3 & 6 & 0.00 & 1 \\
T + S + A & 4742.1 & 6 & 22.8 & 0 \\
\hline
\end{tabular}

sex was unimportant (Adélie: $\chi^{2}=0, \mathrm{p}=1$; gentoo: $\chi^{2}=0.75, p=0.39$ ) and it was therefore dropped from the final models (Table 3 ).

In the final models, the presence of a random effect due to individuals on the intercept suggested that some animals routinely bred earlier than others. The standard deviation of the random effect for individual Adélie penguins was larger than that for gentoo penguins, indicating larger deviations in CID from the population mean among individual Adélie penguins than for individual gentoo penguins. The random year effect, however, was larger for gentoo penguins, indicating a relatively stronger population response among gentoo penguins that all individuals tracked more closely through time.

\section{Known-age study and individual plasticity}

For both species, the effect of breeding experience provided a better predictor for CID than breeding age (Table 1). Thus, we proceeded with fixed effects that included individual-centered October temperatures, the mean October temperature experienced by each individual, breeding experience, and sex.

Comparisons of models with alternative random effects (Table 2) suggested that CIDs in both species were best characterized by random effects on the intercept due to individual and year of breeding; there was less support for the models with random slopes for each individual. From the best full models (Table 2), likelihoodratio tests indicated that the effect of
Table 2. Pygoscelis adeliae and P. papua. Comparison of random-effects models for Adélie and gentoo penguins. The same fixed-effect structure, as identified in Table 1 for each species, was used for all comparisons. For comparison, the model without random effects (Null) is included. The results from these final models were fit using restricted maximum likelihood (REML). The syntax used for the analysis in R is also presented. AIC: Akaike's information criterion; I: individual; na: not applicable; T: individual-centered October temperatures $\left(x_{i j}-\bar{x}_{j}\right)$; Y: year

\begin{tabular}{|lcccccc|}
\hline $\begin{array}{l}\text { Random } \\
\text { effects }\end{array}$ & R syntax & REML & df & AIC & $\Delta$ AIC & $\begin{array}{c}\text { AIC } \\
\text { weight }\end{array}$ \\
\hline $\begin{array}{l}\text { Adélie } \\
\text { I + Y }\end{array}$ & $(1 \mid \mathrm{I})+(1 \mid \mathrm{Y})$ & -789.1 & 8 & 1594.2 & 0 & 0.72 \\
$\mathrm{I}+\mathrm{T}+\mathrm{Y}$ & $(1 \mid \mathrm{I})+(0+\mathrm{TII})+(1 \mid \mathrm{Y})$ & -789.1 & 9 & 1596.2 & 1.94 & 0.28 \\
T + Y & $(0+\mathrm{T} \mid \mathrm{I})+(1 \mid \mathrm{Y})$ & -806.9 & 8 & 1629.8 & 35.6 & 0 \\
Null & na & -825.7 & 6 & 1663.4 & 69.2 & 0 \\
Gentoo & & & & & & \\
I + Y & $(1 \mid \mathrm{I})+(1 \mid \mathrm{Y})$ & -2116.4 & 8 & 4248.8 & 0 & 0.73 \\
I + T + Y & $(1 \mid \mathrm{I})+(0+\mathrm{T} \mid \mathrm{I})+(1 \mid \mathrm{Y})$ & -2116.4 & 9 & 4250.8 & 2 & 0.27 \\
T + Y & $(0+\mathrm{T} \mid \mathrm{I})+(1 \mid \mathrm{Y})$ & -2122.9 & 8 & 4261.9 & 13 & 0 \\
Null & na & -2352.5 & 6 & 4717.1 & 468.3 & 0 \\
\hline
\end{tabular}


Table 3. Pygoscelis adeliae and P. papua. Best-fit mixedeffects model for clutch initiation date of Adélie and gentoo penguins. The fixed effect of temperature was modeled as within-individual centered $\left(x_{i j}-\bar{x}_{j}\right)$ and as the mean for each individual $\left(\bar{x}_{j}\right)$ to distinguish the effects owing to withinindividual differences and across individual differences. Coefficients for the fixed-effect components are reported as mean \pm SE. Random effect is reported as the SD for each component $($ mean $=0)$

\begin{tabular}{|lrr|}
\hline Coefficient & \multicolumn{1}{c|}{ Adélie } & \multicolumn{1}{c|}{ Gentoo } \\
\hline Fixed effects & & \\
Intercept & $302.58 \pm 3.58$ & $303.13 \pm 3.89$ \\
$\left(x_{i j}-\bar{x}_{j}\right)$ & $-1.78 \pm 0.75$ & $-4.26 \pm 1.13$ \\
$\left(\bar{x}_{j}\right)$ & $-1.03 \pm 1.23$ & $-2.99 \pm 1.30$ \\
Breeding experience & $2.26 \pm 0.69$ & $3.55 \pm 0.55$ \\
Random effects & & \\
Individual & 2.97 & 1.85 \\
Year & 3.79 & 6.34 \\
Residual & 4.09 & 5.29 \\
\hline
\end{tabular}

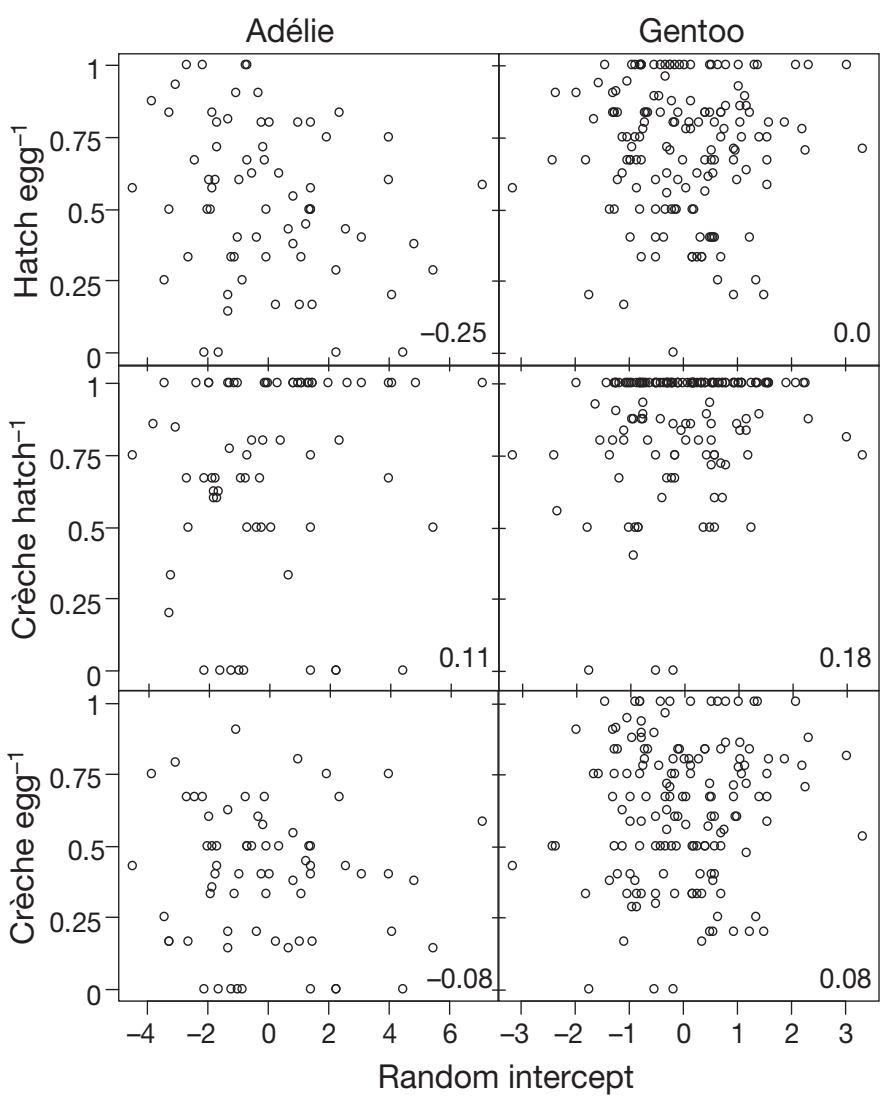

Fig. 5. Pygoscelis adeliae and P. papua. Relationship of the random effect component (intercept) for each individual with respect to its (a) long-term hatch rate, (b) long-term crèching rate, and (c) long-term reproductive success. Data for Adélie penguins are plotted in the left panels and for gentoo penguins in the right panels. Pearson correlation coefficients for each relationship are presented in the lower right corner of each panel
The lack of evidence for different individual rates of responses to changing October temperatures (Table 2) suggested that the within-individual fixed effects had a greater influence on the model than the between-individual fixed effects (Table 3). A reparameterization of the model, as described by van de Pol \& Wright (2009), to test the significance of the between-individual fixed effect, directly confirmed the lack of a between-individual effect (Adélie: $0.68 \pm$ 1.08, $t_{2}=0.63, \mathrm{p}=0.6$; gentoo: $1.27 \pm 0.72 \mathrm{~d}{ }^{\circ} \mathrm{C}^{-1}$, $t_{2}=1.78, \mathrm{p}=0.2$ ), again suggesting that all individuals within each species tended to exhibit similar rates of response to changes in October temperatures.

The individual differences in the timing of CIDs (intercepts), however, were not strongly correlated with estimates of LRS (Fig. 5). The strongest correlation observed was for earlier breeding Adélie penguins to exhibit generally higher hatching rates over their lifetime $\left(\mathrm{r}=-0.25, t_{67}=-2.13, \mathrm{p}=0.04\right)$. However, the apparent advantage of higher long-term hatching rates was not translated into improved LRS as measured by the number of chicks crèched per egg laid. For gentoo penguins, there were no significant correlations between variation in individual CIDs and any metric of LRS (Fig. 5).

\section{DISCUSSION}

\section{Flexible reproductive timing can buffer reproductive success}

In polar environments, where summer breeding seasons are short and food availability can change rapidly, the ability to adjust breeding chronology according to local conditions is considered critical for offspring survival. In a mixed colony of Adélie and gentoo penguins in the Antarctic Peninsula region, we found that plasticity in breeding chronology occurred mainly at the population level, rather than an individual level. Differences between species were also evident, with gentoo penguins exhibiting a greater population-level response in CID to changing temperatures than Adélie penguins. The greater plasticity in CID among gentoo penguins led to an apparent stabilizing effect on mean reproductive success. Specifically, gentoo penguins were capable of advancing $\mathrm{CIDs}$ per ${ }^{\circ} \mathrm{C}$ of warming that was more than twice the amount observed among Adélie penguins. The greater flexibility in mean CIDs among gentoo penguins was associated with a lower CV in mean reproductive success relative to Adélie penguins. This suggests that increased plasticity at a 
population level can help maintain more stable reproductive success, despite negative trends in reproductive success that can occur with delayed breeding. Overall, the greater rate of change in CIDs among gentoo penguins appears to enable the maintenance of relatively high reproductive success across a wide range of environmental conditions. We interpret this as evidence that gentoo penguins are responding more successfully than Adélie penguins to the rapid changes in environmental conditions currently underway in the northern Antarctic Peninsula region (Turner et al. 2005a, Ducklow et al. 2007).

A negative consequence of delayed breeding was evident for some part of reproductive success for both species on a population level, but the effect of changes in the timing of breeding differed by species. For gentoo penguins, later average CIDs were associated with linear declines in population-level hatch and crèche rates, while for Adélie penguins a quadratic relationship best explained the relationship between CID and post-hatch chick survival. The quadratic relationship suggests that, for Adélie penguins, a relatively narrow window for optimal reproductive success does exist, despite the lack of significant trends in hatching rates or in total reproductive success. Forecasts of continued warming for the Antarctic Peninsula region therefore imply earlier CIDs for Adélie penguins and greater rates of posthatch chick loss, while gentoo penguins would be expected to show improvement in both hatch and crèche rates. Expectations of increasing temperatures in the future raise an important question of whether the ability of gentoo penguins to respond to such warming will continue to result in higher reproductive success relative to Adélie penguins.

Previous work (e.g. Perrins 1970, Brinkhof et al. 1993, Dunn 2004) suggests that a delay in breeding typically decreases reproductive success because of the limited time available to provision offspring before local food resources are depleted. Our results are not entirely consistent with this general understanding. At the study site, local food availability must remain sufficiently high during the austral summer for chinstrap penguins Pygoscelis antarctica, historically an abundant penguin species in the region (Trivelpiece et al. 2011), to successfully complete their own reproductive cycle. The diets of Adélie, chinstrap, and gentoo penguins during the breeding season at Admiralty Bay consist mainly of Antarctic krill Euphausia superba (Volkman et al. 1980, W. Z. Trivelpiece unpubl. data), and chinstrap penguins typically initiate their breeding season up to $1 \mathrm{mo}$ after Adélie penguins. Furthermore, annual net and acoustic surveys of Antarctic krill biomass in the Bransfield Strait, which is the main foraging area for penguins from the study location, suggest no seasonal decline in krill biomass between January and February (Reiss et al. 2008). Thus, declines in food availability appear unlikely to be the mechanism contributing to declining reproductive success with delayed breeding. However, physical constraints such as the advance of sea ice or decreases in day length as winter approaches have been implicated in limiting the length of the breeding season (Ainley et al. 1983), such that breeding too late, relative to a local optimum, provides insufficient time to raise chicks successfully. Given such potential physical constraints, breeding success may nonetheless decline with delayed breeding.

For both Adélie and gentoo penguins, the population-level plasticity of CIDs to spring temperatures was stronger than individual-level plasticity. Specifically, we found more support for random effects of individuals and year on the intercept of the mean population response rather than for different slopes that would arise if individuals exhibited different rates of change in CID with respect to October temperatures. The random-effects models, however, indicated that the strength of individual and year effects on the mean CID also differed by species, suggesting a contrasting response of each species to warming condition in the Antarctic Peninsula region. For Adélie penguins, the random effect for year of breeding was smaller than that for gentoo penguins, which is consistent with the greater range of mean population-level CIDs among gentoo penguins across years. However, the random effect due to individuals on the mean CID was larger for Adélie than for gentoo penguins. This suggests that individual Adélie penguins exhibited greater flexibility around their more rigid population mean, while individual gentoo penguins closely tracked their more flexible population mean. Despite the 2 different strategies, the relative timing in breeding of each individual provided no apparent benefit to LRS. This suggests that variation in individual choice about CIDs is secondary to the population-level response to October temperatures among these penguins.

A hallmark of the Pygoscelis spp. breeding biology is the synchronous breeding within dense colonies. Previous work on another highly synchronous colonial breeder, the common guillemot Uria aalge, also found no strong evidence for individual plasticity (Reed et al. 2006). For colonial nesting birds, synchronicity of breeding may provide advantages for chick survival that would be compromised if individuals responded 
differently to ambient conditions than others. Specifically, the period of parental protection among Adélie and gentoo penguins typically lasts 3 to $5 \mathrm{wk}$, after which the chicks gather into crèches and are unattended by adults. Synchronous breeding helps to ensure that chicks are of similar age and size once the crèche period begins. Such similarity in size can be advantageous for chicks faced with avian predators that prefer lone birds (i.e. chicks that enter crèche early or late, relative to the average) or smaller, presumably weaker birds (Young 1994). Also, smaller or lone birds may be more vulnerable to summer storms. For Adélie and gentoo penguins, breeding when your neighbors breed appears to be more important than if individuals adjusted their CID independently.

The observed difference in the ability to adjust CIDs based on October temperatures and subsequent variation in breeding success between gentoo and Adélie penguins may be rooted in species-specific behavioral and energetic constraints. Some long-distance migrants may be less able to adjust breeding chronology according to local conditions relative to sympatric non-migratory species (Møller et al. 2008, Both et al. 2010), primarily because migratory cues in wintering habitats are likely to be uninformative about local nesting conditions. Moreover, the fasting behaviors of Adélie penguins during the breeding season likely place strong energetic constraints on their ability to alter CIDs after arrival when compared with the nonmigratory and continued feeding behaviors of gentoo penguins. A more limited ability to adjust breeding chronology may result in initiating a clutch under conditions that jeopardize reproductive success (Patterson et al. 2003, Büsser et al. 2004, Boersma 2008). Indeed, loss rates of eggs and chicks were roughly twice as high among Adélie penguins as for gentoo penguins; Adélie penguins suffered larger magnitude and more frequent reductions in breeding success than gentoo penguins; and, despite greater individual variation in CID around the population mean, there was no difference in LRS for early versus late breeders. Thus, for Adélie penguins, plasticity in breeding chronology appears to be overridden by energetic constraints that necessitate breeding on a more rigid schedule. For gentoos, the opposite appears to be the case, where variation in reproductive success was more buffered by the ability to respond more strongly to spring temperatures.

Throughout the Antarctic Peninsula region, Adélie and gentoo penguins are currently undergoing rapid changes in the size and distribution of their populations (Forcada et al. 2006, Hinke et al. 2007, Lynch et al. 2008). Adélie-penguin populations have declined throughout the rapidly warming regions of the peninsula, while gentoo-penguin populations have grown and expanded their range southward (Lynch et al. 2008). Much of the decline in the Adélie penguin population is thought to be driven by recent declines in Antarctic krill populations and compounded by changes in overwinter ice conditions in wintering habitats (Trivelpiece et al. 2011). Gentoo penguins, on the other hand, with less strict dependence on krill for food and sea ice as a physical habitat, have proliferated. The data in the present study suggest another factor that may promote the relative success of gentoo penguins in this rapidly changing environment. Specifically, changes in relative breeding chronologies of competing species may alter inter-specific competitive interactions for nesting or food resources (Ahola et al. 2007, Lynch et al. 2012). Segregation of breeding times is thought to represent an important niche separation where Adélie and gentoo penguins breed sympatrically (Trivelpiece et al. 1987). In the northern Antarctic Peninsula region, gentoo populations have historically been quite small relative to Adélie and chinstrap penguin populations, both of which are now in rapid decline (Lynch et al. 2008, Trivelpiece et al. 2011). Changes in competitive interactions that arise when niche separation is diminished can favor species with greater ability to vary the timing of reproduction (e.g. Ahola et al. 2007). Under future conditions of warmer temperatures in the northern Antarctic Peninsula region, the more rapid advance in CIDs among gentoo penguins will shorten or eliminate the difference in timing of breeding between gentoo and Adélie penguins, thus aligning chick provisioning needs of both species. Indeed, in 2008-09, gentoo penguins bred $10 \mathrm{~d}$ earlier than Adélie penguins, the first ever observation of such a reversal in the timing of breeding. An erosion of the difference in the niche space and the potential for increased inter-specific competition for food resources near breeding colonies may further exacerbate stresses on penguin populations in the Antarctic Peninsula region.

\section{Other factors that limit breeding success}

Breeding success of the Pygoscelis spp. penguins can be influenced by numerous factors independent of when the breeding season is initiated, including how variation in the timing of arrival at the colony affects subsequent CIDs, overwinter conditions that dictate the preparedness of adults for subsequent breeding, behavioral constraints of breeders, demo- 
graphic variation in breeding ability, and predation. We briefly discuss each below.

The timing of arrival at the colony, particularly for migratory species, may impact timing of CIDs (Ainley et al. 1983). We have no data on the timing of arrival, largely because data collection usually begins after penguin arrival at our study site. Nonetheless, migratory cues are thought to be physical in nature, such as the changing day length in spring. If such cues are fixed, then variation in arrival dates could be constrained by local conditions near the colony, such as extensive sea ice near the colony (Ainley \& LeResche 1973). In the western Antarctic Peninsula region, rapid warming in the region has reduced the duration of sea ice coverage (Stammerjohn et al. 2008), and by October, the water surrounding the South Shetland Islands are relatively ice-free $(<15 \%$ ice concentration; C. S. Reiss unpubl. data). Thus, physical constraints appear unlikely to have impacted arrival dates at the study colony during the study period.

Alternatively, overwinter conditions could influence the reproductive preparedness of returning adults and compromise the ability of adults to successfully complete a clutch. Vleck \& Vleck (2002) examined the relationship of pre-breeding body condition and subsequent reproductive success in Adélie penguins and reported differences in the body mass of breeding and non-breeding females. Furthermore, they noted a positive relationship between reproductive success and pre-breeding body mass, suggesting that overwinter condition can affect both the decision of whether to breed and the magnitude of reproductive success of individuals that choose to breed. Thus, the higher variation observed in reproductive success of Adélie penguins could be an effect of more variable winter conditions in the northern extent of its range, relative to the conditions experienced by nonmigratory gentoo penguins. Nonetheless, Adélie and gentoo penguins exhibited positive correlations between CIDs and between measures of reproductive success (Hinke et al. 2007), suggesting that ambient conditions during the breeding season exert some control on the reproductive success of both species.

Nest-site fidelity may further constrain reproductive success of Adélie penguins relative to gentoo penguins. Adélie penguins typically breed in the same nesting site year after year, while gentoo penguins more frequently move to new nesting areas, particularly when conditions in old nesting areas are poor (Trivelpiece \& Trivelpiece 1990). Thus, continued foraging and lower nest-site fidelity may provide gentoo penguins greater flexibility to adjust breed- ing chronology to local conditions. The energetic constraints and stronger nest-site fidelity of male Adélie penguins increase the chances of breeding when and where environmental conditions, like heavy snowfall in years with colder spring temperatures, tend to decrease survival rates of offspring. Though we have not analyzed data on snowfall in the present study, mainly because measuring snowfall in windy environments is fraught with difficulty, we note that the poorest reproductive success measured for both species in the present study occurred during years with abnormally high snowfall (W. Z. Trivelpiece pers. obs.). In these years (2007-08 and 2009-10), CIDs were among the latest recorded for both species and hatch rates were among the worst.

Finally, among penguins, breeding success can be affected by intrinsic demographic variation in breeding ability (Ainley et al. 1983, Polito et al. 2010), nest abandonment (Davis 1982), and depredation of offspring and parents (Davis \& McCaffrey 1986, Emslie et al. 1995, Ainley et al. 2005). These factors are undoubtedly important at our study site. Accounting for such effects and relating them to the strong effects of environmental conditions on CIDs provides a promising route for future research.

\section{Climate change and the future of penguin populations}

Flexibility in reproductive chronology that can maintain stable hatching rates is of increased importance given the rapid pace of environmental change in the Antarctic Peninsula ecosystem (Meredith \& King 2005, Turner et al. 2005a,b, Stammerjohn et al. 2008, Thomas et al. 2008). Ongoing trends in air temperature (Turner et al. 2005a) in the region will affect the breeding chronology (Lynch et al. 2012) and, consequently, the reproductive success of Adélie and gentoo penguins, with warming trends leading primarily to earlier breeding. An important consequence of the different responses of these 2 species to changing October temperatures is the likely erosion of niche separation of Adélie and gentoo penguins, with the more stable reproductive success of gentoo penguins potentially contributing to ongoing divergent trends in the population sizes of Adélie and gentoo penguins. As noted by Stearns (1989), a more flexible response to environmental conditions can improve fitness. The observed differences in CIDs and reproductive success of gentoo and Adélie penguins, combined with their divergent population trends in the Antarctic Peninsula region, support this assertion. 
Acknowledgements. We thank the numerous researchers who have collected the long-term data sets used here. Data on monthly meteorology were obtained from the Russian Federation National Antarctic Data Center (NADC) at the Arctic and Antarctic Research Institute (AARI). We gratefully acknowledge funding from the National Science Foundation (NSF; grant \#1016936) and the Lenfest Oceans Program of the Pew Charitable Trusts. Comments from M. Goebel, D. Kinzey, G. Kooyman, H. Lynch, G. Watters, and anonymous reviewers improved this manuscript.

\section{LITERATURE CITED}

Ahola MP, Laaksonen T, Eeva T, Lehikoinen L (2007) Climate change can alter competitive relationships between resident and migratory birds. J Anim Ecol 76:1045-1052

Ainley DG, LeResche RE (1973) The effects of weather and ice conditions on breeding in Adélie penguins. Condor 75:235-255

Ainley DG, LeResche RE, Sladen WJL (1983) Breeding biology of the Adélie penguin. University of California Press, Berkeley, CA

Ainley DG, Ballard G, Karl BJ, Dugger KM (2005) Leopard seal predation rates at penguin colonies of different size. Antarct Sci 17:335-340

Bates D, Maechler M, Bolker B (2011) lme4: linear mixedeffects models using $\mathrm{S} 4$ classes. $\mathrm{R}$ package version 0.99375-39. R Foundation for Statistical Computing, Vienna. http://CRAN.R-project.org/package=lme4

Boersma PD (2008) Penguins as marine sentinels. Bioscience 58:597-607

Both C, van Turnhout CA, Bijlsma RG, Siepel H, van Strien AJ, Froppen RP (2010) Avian population consequences of climate change are most severe for long-distance migrants in seasonal habitats. Proc R Soc Lond B Biol Sci 277:1259-1266

Brinkhof MWG, Cavé AJ, Hage FJ, Verhulst S (1993) Timing of reproduction and fledging success in the coot Fulica atra: evidence for a causal relationship. J Anim Ecol 62: 577-587

> Büsser C, Kahles A, Quillfeldt P (2004) Breeding success and chick provisioning in Wilson's storm petrel Oceanites oceanicus over seven years: frequent failures due to food shortage and entombment. Polar Biol 27:613-622

Clausen A, Pütz K (2003) Winter diet and foraging range of gentoo penguins (Pygoscelis papua) from Kidney Cove, Falkland Islands. Polar Biol 26:32-40

Davis LS (1982) Timing of nest relief and its effects on breeding success in Adélie penguins (Pygoscelis adeliae). Condor 84:178-183

Davis LS, McCaffrey FT (1986) Survival analysis of eggs and chicks of Adélie penguins (Pygoscelis adeliae). Auk 103: 379-388

> Ducklow HW, Baker K, Martinson DG, Quetin LB and others (2007) Marine pelagic ecosystems: the West Antarctic Peninsula. Philos Trans R Soc Lond B Biol Sci 362:67-94

> Dunn P (2004) Breeding dates and reproductive performance. Adv Ecol Res 35:69-97

Emslie SD, Karnovsky N, Trivelpiece W (1995) Avian predation at penguin colonies on King George Island, Antarctica. Wilson Bull 107:317-327

> Forcada J, Trathan PN, Reid K, Murphy EJ, Croxall JP (2006) Contrasting population changes in sympatric penguin species in association with climate warming. Glob Change Biol 12:411-423

Hinke JT, Salwicka K, Trivelpiece SG, Watters GM, Trivelpiece WZ (2007) Divergent responses of Pygoscelis penguins reveal a common environmental driver. Oecologia 153:845-855

> Loeb V, Siegel V, Holm-Hansen O, Hewitt R, Fraser W, Trivelpiece W, Trivelpiece S (1997) Effects of sea-ice extent on krill or salp dominance on the Antarctic food web. Nature 387:897-900

Lynch HJ, Naveen R, Fagan WF (2008) Censuses of penguin, blue-eyed shag Phalacrocorax atriceps and southern giant petrel Macronectes giganteus populations on the Antarctic Peninsula, 2001-2007. Mar Ornithol 36: 83-97

Lynch HJ, Fagan WF, Naveen R, Trivelpiece SG, Trivelpiece WZ (2009) Timing of clutch initiation in Pygoscelis penguins on the Antarctic Peninsula: towards an improved understanding of off-peak census correction factors. CCAMLR Sci 16:149-165

Lynch HJ, Fagan WF, Naveen R, Trivelpiece SG, Trivelpiece WZ (2012) Differential advancement of breeding phenology in response to climate may alter staggered breeding among sympatric pygoscelid penguins. Mar Ecol Prog Ser 454:135-145

Meredith MP, King JC (2005) Rapid climate change in the ocean west of the Antarctic Peninsula during the second half of the 20th century. Geophys Res Lett 32:L19604 doi:10.1029/2005GL024042

Møller AP, Rubolini D, Lehikoinen A (2008) Populations of migratory bird species that did not show a phenological response to climate change are declining. Proc Natl Acad Sci USA 105:16195-16200

Monrós JS, Belda EJ, Barba E (1998) Delays of the hatching dates in great tits Parus major: effects on breeding performance. Ardea 86:213-220

Morris WF, Doak DF (2004) Buffering of life histories against environmental stochasticity: accounting for spurious correlation between the variabilities of vital rates and their contributions to fitness. Am Nat 163:579-590

Patterson DL, Easter-Pilcher AL, Fraser WR (2003) The effects of human activity and environmental variability on long-term changes in Adélie penguin populations at Palmer Station, Antarctica. In: Huiskes AHL, Gieskes WWC, Rozema J, Schorno RML, van der Vies SM, Wolf W (eds) Antarctic biology in a global context. Backhuys Publishers, Leiden, p 301-307

> Perrins CM (1970) The timing of birds' breeding seasons. Ibis 112:242-255

Polito MJ, Miller AK, Trivelpiece SG, Trivelpiece WZ (2010) Maturation increases early reproductive investment in Adélie Penguins Pygoscelis adeliae. Ibis 152:38-47

R Development Core Team (2010) R: a language and environment for statistical computing. R Foundation for Statistical Computing, Vienna. www.R-project.org

Reed TE, Wanless S, Harris MP, Frederiksen M, Kruuk LEB, Cunningham EMA (2006) Responding to environmental change: plastic responses vary little in a synchronous breeder. Proc R Soc Lond B Biol Sci 273:2713-2719

> Reiss CS, Cossio AM, Loeb V, Demer DA (2008) Variations in the biomass of Antarctic krill (Euphausia superba) around the South Shetland Islands, 1996-2006. ICES J Mar Sci 65:497-508

Schofield O, Ducklow HW, Martinson DG, Meredith MP, Moline MA, Fraser WR (2010) How do polar marine 
ecosystems respond to climate change? Science 328: 1520-1523

Stammerjohn SE, Martinson DG, Smith RC, Iannuzzi RA (2008) Sea-ice in the western Antarctic Peninsula region: spatio-temporal variability from ecological and climate change perspectives. Deep-Sea Res II 55: 2041-2058

Stearns SC (1989) The evolutionary significance of phenotypic plasticity. BioScience 39:436-445

Tanton JL, Reid K, Croxall JP, Trathan PN (2004) Winter distribution and behavior of gentoo penguins Pygoscelis papua at South Georgia. Polar Biol 27:299-303

Thomas ER, Marshall GJ, McConnell JR (2008) A doubling in snow accumulation in the western Antarctic Peninsula since 1850. Geophys Res Lett 35:L01706 doi.10.1029/ 2007GL032529

Trivelpiece WZ, Trivelpiece SG (1990) Courtship period of Adélie, gentoo, and chinstrap penguins. In: Davis LS, Darby JT (eds) Penguin biology. Academic Press, San Diego, CA, p 113-127

Trivelpiece WZ, Trivelpiece SG, Volkman NJ (1987) Ecological segregation of Adélie, gentoo, and chinstrap penguins at King George Island, Antarctica. Ecology 68: 351-361

Trivelpiece WZ, Hinke JT, Miller AKM, Reiss CS, Trivelpiece SG, Watters GM (2011) Variability in krill biomass links harvesting and climate warming to penguin popu-

Editorial responsibility: Rory Wilson,

Swansea, UK lation changes in Antarctica. Proc Natl Acad Sci USA 108:7625-7628

Turner J, Colwell SR, Marshall GJ, Lachlan-Cope TA and others (2005a) Antarctic climate change during the last 50 years. Int J Climatol 25:279-294

Turner J, Lachlan-Cope T, Colwell S, Marshall GJ (2005b) A positive trend in Western Antarctic Peninsula precipitation over the last 50 years reflecting regional and Antarctic-wide atmospheric circulation changes. Ann Glaciol 41:85-91

van de Pol M, Wright J (2009) A simple method for distinguishing within- versus between-subject effects using mixed models. Anim Behav 77:753-758

Visser ME (2008) Keeping up with a warming world: assessing the rate of adaptation to climate change. Proc R Soc Lond B Biol Sci 275:649-659

> Vleck CM, Vleck D (2002) Physiological condition and reproductive consequences in Adélie penguins. Integr Comp Biol 42:76-83

> Volkman NJ, Pressler P, Trivelpiece W (1980) Diets of Pygoscelid penguins at King George Island, Antarctica. Condor 82:373-378

Young E (1994) Skua and penguin: predator and prey. Cambridge University Press, Cambridge

Zuur AF, Ieno EN, Walker NJ, Saveliev AA, Smith GM (2009) Mixed effects models and extensions in ecology with R. Springer, New York, NY

Submitted: December 22, 2010; Accepted: January 27, 2012 Proofs received from author(s): April 19, 2012 